\title{
Flight Metabolism in Panstrongylus megistus (Hemiptera: Reduviidae): the Role of Carbohydrates and Lipids
}

\author{
Lilián E Canavoso, Raúl Stariolo*, Edilberto R Rubiolo/+ \\ Departamento de Bioquímica Clínica, Facultad de Ciencias Químicas, Universidad Nacional de Córdoba, H. Torre y M. Allende, \\ CP 5000, Ciudad Universitaria, Córdoba, Argentina *Coordinación Nacional de Control de Vectores, Ministerio de Salud de la \\ Nación, Córdoba, Argentina
}

\begin{abstract}
The metabolism of lipids and carbohydrates related to flight activity in Panstrongylus megistus was investigated. Insects were subjected to different times of flight under laboratory conditions and changes in total lipids, lipophorin density and carbohydrates were followed in the hemolymph. Lipids and glycogen were also assayed in fat body and flight muscle. In resting insects, hemolymph lipids averaged $3.4 \mathrm{mg} / \mathrm{ml}$ and significantly increased after $45 \mathrm{~min}$ of flight $(8.8 \mathrm{mg} / \mathrm{ml}, P<0.001)$. High-density lipophorin was the sole lipoprotein observed in resting animals. A second fraction with lower density corresponding to low-density lipophorin appeared in insects subjected to flight. Particles from both fractions showed significant differences in diacylglycerol content and size. In resting insects, carbohydrate levels averaged $0.52 \mathrm{mg} / \mathrm{ml}$. They sharply declined more than twofold after $15 \mathrm{~min}$ of flight, being undetectable in hemolymph of insects flown for $45 \mathrm{~min}$. Lipid and glycogen from fat body and flight muscle decreased significantly after 45 min of flight. Taken together, the results indicate that P. megistus uses carbohydrates during the initiation of the flight after which, switching fuel for flight from carbohydrates to lipids.
\end{abstract}

Key words: Chagas - Panstrongylus megistus - flight - lipids - carbohydrates

Triatominae insects (Hemiptera: Reduviidae) are vectors of the parasite Trypanosoma cruzi, the causative agent of Chagas disease. The disease represents a serious health hazard in many countries, and current estimations indicate that about $25 \%$ of Latin American population is at risk of infection (WHO 1991, Guzman-Bracho 2001).

Triatomine insects have two mechanisms of dispersal: passive or active (Neghme et al. 1960, Soler et al. 1969, Schofield 1994). Active dispersal by flight performed by winged adults is highly temperature dependent and has been associated with the poor nutritional status of the vectors in response to habitat destruction, increasing bug population density, host death as well as restricted access to blood sources (Sjogren \& Ryckman 1966, Schofield 1980, Lehane \& Schofield 1982, Noireau \& Dujardin 2001).

One of the most important Chagas transmitters in South America is Panstrongylus megistus, which is widely distributed throughout Central Brazil and Eastern Paraguay. Although P. megistus is principally a nondomestic vector, adults fly into houses at night, colonizing peridomestic environments, even invading the houses where Triatoma infestans, the main domestic vector, has been eliminated by control measures (Schofield 1994).

Flight is one of the most remarkable activities performed by insects and requires a high-energy turnover (Candy et al. 1997). Insect neuropeptides, the adipokinetic hormones (AKHs)/ hypertrehalosemic hormones, play an

Financial support: SECyT-UNC (ERR) and Fundación Antorchas (LEC)

${ }^{+}$Corresponding author. Fax: +54-351-433.3048. E-mail: erb@mail.fcq.unc.edu.ar

Received 25 April 2003

Accepted 10 September 2003 important role in the coordination and integration of the mechanism of the fuel supply during insect flight (Candy et al. 1997, Goldsworthy et al. 1997).

From a biochemical viewpoint, lipids represent the main source of energy for the flight muscle in long-term-flying insects (Chino 1997, Ryan \& Van der Horst 2000). However, some species can use carbohydrate reserves or proline (Candy et al. 1997, Gäde \& Auerswald 2002). Lipids are mainly stored in the fat body as triacylglycerols. They are transported in the hemolymph as diacylglycerols by highdensity lipophorin (HDLp), the main insect lipoprotein. Shortly after the onset of flight, AKH is released from the neurosecretory cells of the corpus cardiacum and stimulates the loading of lipophorin with diacylglycerol at the fat body (reviewed by Ryan \& Van der Horst 2000, Arrese et al. 2001). Consequently, lipophorin becomes larger and heterogeneous, generating a low-density lipophorin particle (LDLp). After unloading of diacylglycerol to the flight muscle, which acts as fuel by intracellular oxidation of their fatty acids, LDLp is recycled generating the original particle found in resting insects, HDLp (Chino 1997, Canavoso et al. 2001).

Little is known about the metabolic pathways involved during the flight of triatomine bugs. Ward et al. (1982) reported that flight in Rhodnius prolixus induced both changes in the muscle content of lipid and glycogen and the increase of hemolymph lipid. However, no changes in the hemolymph carbohydrate in insects flown for a short time were observed. On another hand, by employing fat bodies from starved triatomine bugs, we previously reported that AKH promoted generation of LDLp, the lipoprotein responsible for the delivery of lipids during flight (Canavoso \& Rubiolo 1995).

The present study was directed to elucidate the biochemical basis involved during the flight activity of triatomine bugs, by stimulating the flight in P. megistus 
under laboratory conditions. The changes in lipids and carbohydrates found in hemolymph, fat body and flight muscle after different times of flight support the hypothesis that $P$. megistus uses carbohydrates during the initiation of the flight after which, switching fuel from carbohydrates to lipids.

\section{MATERIALS AND METHODS}

Chemicals - The enzymatic kit for acylglycerols was from Boehringer. Anti-rabbit immunoglobulin conjugated to horseradish peroxidase, $o$-phenylenediamine and, microtiter plates were obtained from Sigma Chemical Company. Lipid standards were from Avanti Polar Lipids. Thin layer chromatography (TLC) silica gel plates were purchased from Merck. All other chemicals used in the experiments were analytical grade.

Insects - Insects from a colony of P. megistus established from 1995 in our insect facility were fed every two weeks on hen blood. Adults of both sexes (in approximately equal number) were fed at day 5 after final ecdysis and those with similar weights were selected for the experiments. Insects were maintained at $28^{\circ} \mathrm{C} \pm 1^{\circ} \mathrm{C}$, 60-70\% humidity, 8:16 h light:dark photoperiod cycle and used 15 days post-feeding (Canavoso \& Rubiolo 1993). The average of body weight of the insects at the time of flight experiments was $217 \pm 25 \mathrm{mg}$.

Flight experiments - Experiments were performed in a thermostat-controlled room $\left(30^{\circ} \mathrm{C}, 70 \%\right.$ humidity $)$ with some modifications according to Gringorten and Friend (1979). Specifically, insects were tethered by a loop of wire round the junction of head and thorax and with their tarsi free of any surface contact were suspended in front of an air stream. Flight was stimulated among these restrained insects for $15 \mathrm{~min}$ and $45 \mathrm{~min}$. If the beating of their wings ceased, they were re-stimulated by contacting momentarily their tarsi with a substrate. Those insects that interrupted the flight more than twice and for periods longer than $15 \mathrm{~s}$ were discarded. Insects without flight stimulus were used as controls and they are designated as "resting insects" or "non-stimulated insects".

Hemolymph and tissues sampling - Hemolymph from flown and resting insects was obtained on ice-cold microtubes as previously described (Canavoso \& Rubiolo 1993). Hemolymph was then centrifuged at $10,000 \mathrm{~g}$ for 10 min at $4^{\circ} \mathrm{C}$ to remove hemocytes, fractionated with protease inhibitors and stored at $-70^{\circ} \mathrm{C}$ until the assays were performed. For the carbohydrate determination, the hemolymph was collected only with $10 \mathrm{mM} \mathrm{Na}{ }_{2}$ EDTA and immediately used (Canavoso \& Rubiolo 1998).

Preliminary assays demonstrated that lipid and carbohydrate levels measured on individual insects were not significantly different from those obtained from pooled hemolymph. Thus, to ensure enough volume to perform all the chemical assays, hemolymph from five groups with two insects each were employed to analyze resting or flight conditions.

After hemolymph collection, insects were carefully dissected on ice-cold saline solution $(150 \mathrm{mM} \mathrm{NaCl} ; 20$ $\mathrm{mM} \mathrm{KCl}$ ). The tissues (fat body and flight muscles) were washed, dried on filter paper, weighed with $\pm 0.01 \mathrm{mg}$ and processed individually for lipid and glycogen content.
Chemical assays in tissues and hemolymph - Lipids and glycogen from fat body and flight muscle were separated according to Van Handel (1965). Lipids and carbohydrates present in tissues and hemolymph were quantified as described by Frings and Dunn (1970) and Scott and Melvin (1953), respectively. Protein concentration was determined by Bradford assay (1976). Lipophorin titer was assayed by indirect ELISA assay, using an antiserum against purified lipophorin from $P$. megistus (Canavoso \& Rubiolo 1995, 1998).

Analysis by TLC - Hemolymph lipids from flown or resting insects were extracted (Bligh \& Dyer 1959) and analyzed by TLC on silica gel plates, using hexane/ethyl acetate $(9: 1 \mathrm{v} / \mathrm{v})$ as developing system. Lipids were detected by spraying the plates with $10 \% \mathrm{H}_{2} \mathrm{SO}_{4} / 0.04 \mathrm{M}$ $\left(\mathrm{NH}_{4}\right)_{6} \mathrm{Mo}_{7} \mathrm{O}_{24} .4 \mathrm{H}_{2} \mathrm{O} / 3 \mathrm{mM} \mathrm{Ce}\left(\mathrm{SO}_{4}\right)_{2}$ and heating to $200^{\circ} \mathrm{C}$. Quantification of sn-1,2 (2,3)- and sn-1,3diacylglycerols was performed by densitometry (Bio Rad GS 670 and Kodak Digital Science), using internal standards (Canavoso et al. 1998).

Lipophorin isolation - HDLp and LDLp were isolated by $\mathrm{KBr}$ density gradient ultracentrifugation, using $1.5 \mathrm{ml}$ of hemocyte-free hemolymph collected from flown or resting insects (about 120-150 insects for each condition) (Canavoso \& Rubiolo 1995). After centrifugation the tube contents were fractionated from the top into $1 \mathrm{ml}$ fractions. In each fraction the density, using refractive index, and the absorbance at $280 \mathrm{~nm}$ were determined. Lipophorins were dialyzed against phosphate buffered saline (PBS) at $\mathrm{pH} 7.4$ and their content in acylglycerols was determined by enzymatic methods as previously reported (Canavoso \& Rubiolo 1995).

Electron microscopy - Lipophorins isolated by ultracentrifugation were dialyzed against a volatile buffer (0.125 M ammonium acetate, $2.6 \mathrm{mM}$ ammonium carbonate and $0.26 \mathrm{mM} \mathrm{Na}_{2}$ EDTA, pH 7.4) and applied to Formvarcarbon-coated grids. For negative staining studies, $20 \mu \mathrm{l}$ of the lipophorin solution were applied to the support film, incubated for $5 \mathrm{~min}$, dried, rinsed with redistilled water and contrasted with $2 \%$ sodium phosphotungstate, $\mathrm{pH}$ 5.5 (Forte \& Nordhausen 1986). Grids were examined using a JEOL 1200 EX electron microscope.

Statistical analyses - Statistical tests were performed using GraphPad InStat (GraphPad Software, San Diego, CA). Unless otherwise stated, results are reported as mean \pm SEM of 3 independent experiments. $\mathrm{P}<0.05$ was considered as a significant difference between means. All determinations were done in duplicate.

\section{RESULTS}

Most insects were able to perform stationary flight for 45 min (85-92\%). However, P. megistus exhibited the potential for sustained and continuous wing beat for periods greater than $1 \mathrm{~h}$. Preliminary assays revealed no significant differences between sexes in any of the parameters measured. Therefore, males and females in approximately equal number were used.

The lipids in the hemolymph, which in resting insects averaged $3.4 \mathrm{mg} / \mathrm{ml}$, showed an increase at both times of flight analyzed, with the levels after $45 \mathrm{~min}$ of flight significantly greater than those at rest $(8.8 \mathrm{mg} / \mathrm{ml}, P<$ 
0.001) (Fig. 1). The analysis of hemolymph lipids by TLC demonstrated that flight induced an increase in the diacylglycerol fraction, affecting principally $s n-1,2(2,3)$ diacylglycerols. Densitometry analysis showed that after 45 min of flight $s n$-1,2 (2,3)-diacylglycerol represented 79.3 $\pm 2.3 \%$ of the total diacylglycerol fraction, whereas in resting insects it represented about $57.8 \pm 1.5 \%$ (Fig. 2). On another hand, carbohydrate concentration in the hemolymph of non-stimulated insects reached a mean of $0.52 \mathrm{mg} / \mathrm{ml}$. The carbohydrate levels sharply declined more than twofold after $15 \mathrm{~min}$ of flight and were undetectable, using the anthrone reaction, in hemolymph of insects flown for $45 \mathrm{~min}$ (Fig. 1). The levels of hemolymph lipids and carbohydrates observed in insects flown for $45 \mathrm{~min}$ were restored to resting values at $24 \mathrm{~h}$ after flight termination (results not shown).

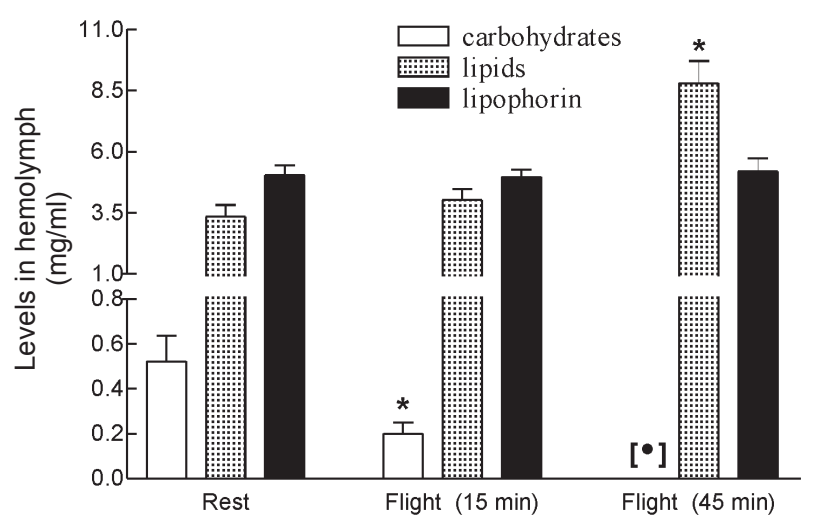

Fig. 1: levels of carbohydrates, total lipids, and total lipophorin (as high-density lipophorin + low-density lipophorin) in hemolymph of Panstrongylus megistus at rest and after flight activity. [•]: levels of carbohydrates undetected after flight. For the conditions analyzed, hemolymph from five groups with two insects each was assayed. Chemical determinations were done in duplicate. Data represent the mean \pm SEM of three independent experiments. Lipids after flight $(45 \mathrm{~min}, * P<0.001)$ vs lipids at rest; carbohydrates after flight $(15 \mathrm{~min}, * P<0.001)$ vs carbohydrates at rest
The amount of lipid and glycogen stored in fat body and flight muscle of resting P. megistus and the changes that occurred in the tissues during flight were also analyzed (Fig. 3A-B). At both times of flight studied, a significant decrease in lipid and glycogen from flight muscle was observed compared with values from resting insects (Fig. 3A). In fat body only glycogen declined after insects were flown for $15 \mathrm{~min}$. However, the decrease in lipid and glycogen content from fat body was significant in insects flown for $45 \mathrm{~min}$ (Fig. 3B).

Since flight induced a significant increase in hemolymph lipids, the changes in density and levels of the main hemolymph lipid carrier, the HDLp were also investigated. When hemolymph of resting $P$. megistus was subjected to ultracentrifugation, a sole fraction of HDLp with a density range of $1.127-1.131 \mathrm{~g} / \mathrm{ml}$ was observed. After 45 min of flight, however, two lipophorin fractions with densities ranging between $1.125-1.131 \mathrm{~g} / \mathrm{ml}$ and 1.078 $1.089 \mathrm{~g} / \mathrm{ml}$ (Fig. 4A) were isolated from the hemolymph. The first fraction corresponded to HDLp, whereas the fraction with lower density corresponded to LDLp. The sum of absorbance at $280 \mathrm{~nm}$ of HDLp and LDLp density gradient of flown insects was similar to the HDLp amount of resting insects. In addition, HDLp levels measured by ELISA in resting insects ranged between $4.1-5.7 \mathrm{mg} / \mathrm{ml}$ and the total amount of lipophorin (as HDLp + LDLp) did not change significantly in the stimulated insects (Fig. 1). Taken both observations together, it can be surmised that LDLp was originated by partial conversion of HDLp after the hyperlipaemic effect of flight.

Electron micrographs of the two-lipophorin fractions isolated from P. megistus after flight showed that HDLp particles were a homogeneous population of lipoproteins, ranging in size from $10-14 \mathrm{~nm}$. These particles were similar to HDLp particles found in resting insects (Fig. 4 B). In contrast, the LDLp fraction showed great heterogeneity in size with a predominance of particles larger than $20 \mathrm{~nm}$ (Fig. 4C).
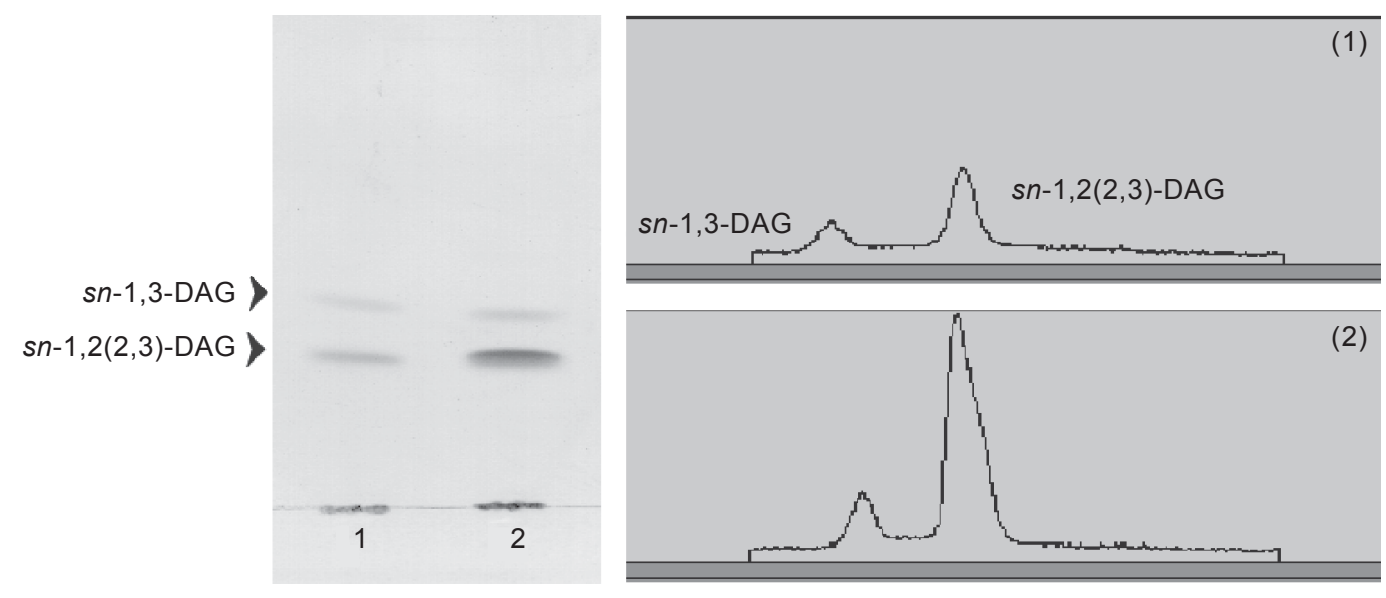

Fig. 2: thin layer chromatography on silica gel (hexane/ethyl acetate, 9:1 v/v) of total lipid extracts of hemolymph from Panstrongylus megistus at rest (1) and after $45 \mathrm{~min}$ of flight (2). Head arrows indicate the positions for $s n-1,2(2,3)$ - and $s n-1,3$ diacylglycerol (DAG). Figure also shows the densitometry profile for $s n-1,2(2,3)-$ DAG and $s n-1,3-D A G$. Results are representative of three independent experiments. 
A

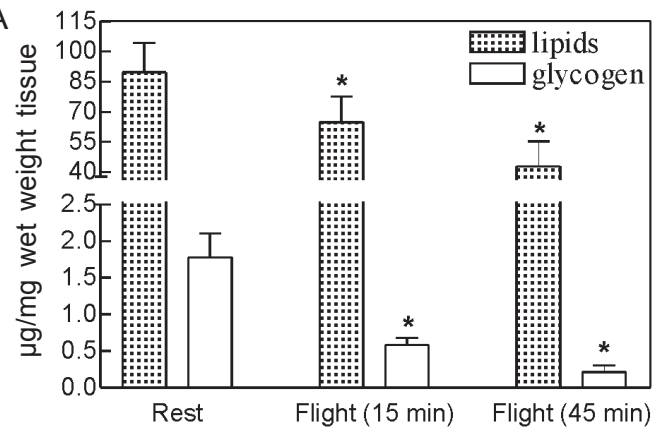

B

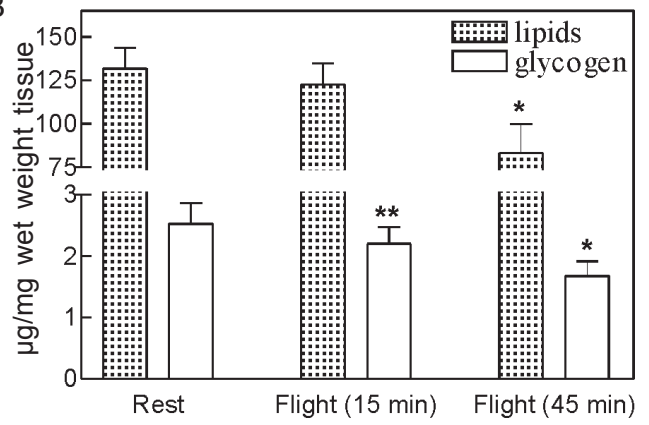

Fig. 3: content of lipid and glycogen in flight muscle (A) and fat body (B) of Panstrongylus megistus at rest or after flight activity. Tissues were processed individually as described under Materials and Methods and chemical determinations were done in duplicate. Data represent the mean \pm SEM $(\mathrm{n}=10)$ of three independent experiments. Flight muscle: lipids and glycogen after flight vs resting condition $(* P<$ $0.001)$. Fat body: lipids after flight $(45 \mathrm{~min}, * P<0.001)$ vs lipids at rest; glycogen after flight $(15 \mathrm{~min}, * * P<0.05 ; 45 \mathrm{~min}, * P<0.001)$ vs resting condition

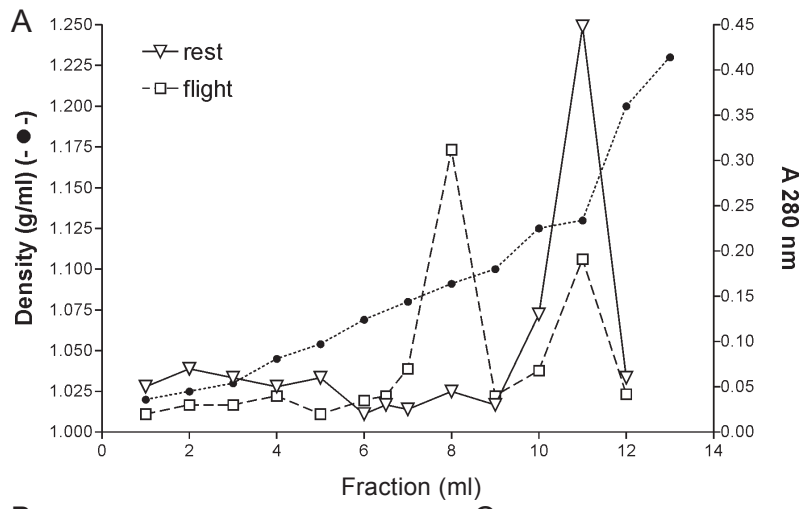

B

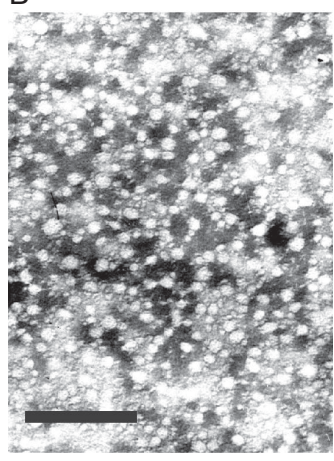

Fig. 4A: density gradient ultracentrifugation profile of lipophorins from insects at rest $(-\nabla-)$ and after 45 min of flight $(-\square-)$. About $1.5 \mathrm{ml}$ of hemocyte-free hemolymph were loaded in each gradient as described in Materials and Methods. Absorbance at $280 \mathrm{~nm}$ was determined in the fractions previously diluted with $150 \mathrm{mM} \mathrm{NaCl}$ (1:6). low-density lipophorin (LDLp) was located within fractions 7-8, whereas high-density lipophorin (HDLp) was found in the fractions 10-11. Data represent the mean \pm SEM of two independent experiments; B-C: electron micrographs of negatively stained particles corresponding to HDLp (B, rest) and LDLp (C, flight). Bars represent $200 \mathrm{~nm}$ and $100 \mathrm{~nm}$, respectively.

The proportion of acylglycerols in HDLp from both resting and flight stimulated insects did not change significantly ( $48.5 \pm 2.1 \%$ vs. $50.3 \pm 1.6 \%$ respectively; $\mathrm{n}=$ 4). This percentage, however, was significantly changed in LDLp with acylglycerols representing about $80 \%$ of their structure $(79.1 \pm 4.6 \%, P<0.001, \mathrm{n}=4)$.

\section{DISCUSSION}

The experiments described in this work suggest that P. megistus has the potential to perform sustained flight for a period longer than $1 \mathrm{~h}$. This finding is in agreement with reports of nocturnal flights by P. megistus (Schofield 1994). Similar flight activities were described for $R$. prolixus (Gringorten \& Friend 1979, Ward et al. 1982), T. infestans (Schofield et al. 1992) and more recently, for T. sordida and T. guasayana (Noireau \& Dujardin 2001).

Ward et al. (1982) had previously suggested the involvement of lipids as the energy source used by triatomine bugs during their dispersal by flight. The presence of lipid droplets in flight muscle has been reported in at least three vectors of Chagas disease including T. infestans, $R$. prolixus, and D. maxima (Ward et al. 1982, Cavagnari et al. 2000). In addition, studies in $R$. prolixus and $T$. infestans showed that lipid and glycogen stores in flight muscle declined with the time of flight, whereas no significant differences in the amount of abdomen lipids were found in flown and resting insects (Ward et al. 1982). In our experiments using P. megistus, we also observed the decrease of flight muscle lipids at both times of flight analyzed. However, after $45 \mathrm{~min}$ of flight, lipid stores in fat body also declined to $60 \%$ of the amount present in resting insects (Fig. 3). In the hemolymph, lipid levels increased significantly with the time of flight, whereas carbohydrates levels decreased until they were undetectable after 45 min of flight (Fig.1).

In agreement with in vitro studies using fat bodies and exogenous AKH (Chino et al. 1989, Canavoso \& Rubiolo 1995) and with in vivo studies using AKH injection (Lum \& Chino 1990), we reported that after flight, the changes in the hemolymph lipids were associated with the generation of LDLp particles. The LDLp particles loaded with a higher content of acylglycerol showed heterogeneous size with a predominance of the particles being $22 \mathrm{~nm}$ in size, whereas HDLp exhibited small size and homogeneity (Fig. 4B-C). Similar observations were obtained in Locusta migratoria by Chino (1997). However, the results obtained using $P$. megistus are unique in that flight promoted the partial interconversion of lipophorins and therefore, both HDLp and LDLp were observed. The partial lipophorin interconversion has been reported in in 
vitro AKH studies using fat bodies from L. migratoria or M. sexta, two species adopted as models for flight-induced lipophorin conversions (Chino et al. 1989, Van Heusden et al. 1984, 1987, Van der Horst et al. 1988). Currently, $P$. megistus is the first species in which flight elicits partial formation of LDLp.

In our experiments, the partial lipophorin interconversion is demonstrated by the fact that the total amount of lipophorin (as HDLp and LDLp) in the hemolymph of flown insects was similar to the HDLp amount found in resting insects, as determined by the absorbance measurements in the $\mathrm{KBr}$ density gradient (Fig. 4A). In addition, when tested by ELISA, no changes were observed on the total lipophorin levels after flight (Fig. 1). Altogether, the results are also in agreement with the role of lipophorin as reusable shuttle, which during flight moves lipids from one tissue to another without being degraded and without biosynthesis of new lipophorin particles (Canavoso et al. 2001).

The fact that flight in P. megistus induces the partial formation of LDLp presently remains uncertain. Chino et al. (1992) suggested that during flight, the interconversion of lipophorins could only occur when a large fat body diacylglycerol pool is present, besides the triacylglycerol pool as its precursor source. In a comparative study about lipid composition in fat body from different species of triatomine bugs, we found that $P$. megistus had both, a large amount of lipids (where triacylglycerol represented about $54.2 \%$ of the total neutral lipids) and the largest diacylglycerol pool (37.2\%) (Canavoso et al. 1998). We also reported that HDLp and hemolymph of P. megistus has apolipophorin-III, an essential component for LDLp formation (Canavoso \& Rubiolo 1995). Since the molecular components of the flight-related lipid transport system are present in P. megistus, it appears that the partial interconversion of lipophorin induced by flight is the result of the difference between the duration of continuous flight and distance characteristic of locusts, which can completely transform HDLp to LDLp. For instance, migratory locusts are capable of uninterrupted flight for $10 \mathrm{~h}$, covering more than $200 \mathrm{~km}$ (Ryan \& Van der Horst 2000). In contrast, $P$. megistus is not a typical flying insect and although we demonstrate its ability for sustained flight longer than $1 \mathrm{~h}$, it is unlikely it would be capable to perform such a prolonged physical exercise. In this context, the partial formation of LDLp in P. megistus can be interpreted as an intermediate metabolic situation in the spectrum of insects that perform long-distance flight and those that are incapable of flight.

It is worthy to note that the amount of hemolymph carbohydrates in P. megistus is considerably smaller than that of insects that use carbohydrates as fuel (Wyatt 1967, Jutsum \& Goldsworthy 1976). In this vector we observed that flight increases the expenditure of carbohydrates: it was shown that a rapid decrease of the hemolymph carbohydrate concentration and the glycogen stores in flight muscle significantly declined during the first $15 \mathrm{~min}$ of flight. After $45 \mathrm{~min}$ of flight, the levels of hemolymph carbohydrates were undetectable and glycogen in flight muscle represented only $10 \%$ of that found in control insects. However, the glycogen in fat body of resting $P$. megistus represents about $3.6 \%$ of the wet weight of the organ, an amount 4-fold greater than that found in another triatomine, D. maxima (Canavoso \& Rubiolo 1998). Therefore, it is likely that at the onset of flight $P$. megistus uses carbohydrates as fuel, inducing the mobilization of the glycogen stored in fat body.

The increase in lipid levels, as well as the partial metabolic conversion of lipophorin generating LDLp particles with the concomitant decrease of carbohydrates in hemolymph allowed speculation about the main events during flight of triatomine bugs. It is hypothesized that $P$. megistus would use carbohydrates during initiation of flight (first 10-15 min) after which, the switching of fuel for flight from carbohydrates to lipids takes place. Thereafter, lipids become the main fuel for sustained flight, likely by an AKH-mediated mechanism.

The hyperlipaemic effect observed in insects subjected to flight, as well as the lipid mobilization from fat body using an in vitro system and exogenous AKH (Canavoso \& Rubiolo 1995) suggest the presence of an AKH-like factor in P. megistus. In fact, AKH has been demonstrated in most of insects, and triatomine bugs should not be an exception.

\section{ACKNOWLEDGEMENTS}

To Graciela Truol for technical support in electron microscopy. To James Pennington for critical reading of the manuscript.

\section{REFERENCES}

Aresse EL, Canavoso LE, Jouni ZE, Pennington JE, Tsuchida K, Wells MA 2001. Lipoprotein metabolism in insects: current status and future directions. Insect Biochem Molec Biol 31: 7-17.

Bligh EG, Dyer WJ 1959. A rapid method of total lipid extraction and purification. Can J Biochem Physiol 37: 911917.

Bradford M 1976. A rapid and sensitive method for quantitation of proteins utilizing the principle of protein dye binding. Anal Biochem 72: 248-254.

Canavoso LE, Rubiolo ER 1993. Hemolymphatic components in vectors of Trypanosoma cruzi. Study in several species of the subfamily Triatominae (Hemiptera: Reduviidae). Rev Inst Med Trop São Paulo 35: 123-128.

Canavoso LE, Rubiolo ER 1995. Interconversion of lipophorin particles by adipokinetic hormone in hemolymph of Panstrongylus megistus, Dipetalogaster maximus, and Triatoma infestans (Hemiptera : Reduviidae). Comp Biochem Physiol 112A: 143-150.

Canavoso LE, Rubiolo ER 1998. Metabolic post-feeding changes in fat body and hemolymph of Dipetalogaster maximus (Hemiptera: Reduviidae). Mem Inst Oswaldo Cruz 93: 225-230.

Canavoso LE, Bertello LE, Lederkremer RM, Rubiolo ER 1998. Effect of fasting on the composition of the fat body lipid of Dipetalogaster maximus, Panstrongylus megistus, and Triatoma infestans (Hemiptera: Reduviidae). J Comp Physiol B 168: 549-554.

Canavoso LE, Jouni EJ, Karnas KJ, Pennington JE, Wells MA 2001. Fat metabolism in insects. Annu Rev Nutr 21: 23-46.

Candy DJ, Becker A, Wegener G 1997. Coordination and integration of metabolism in insect flight. Comp Biochem Physiol 117B: 497-512.

Cavagnari BM, Scaraffia PY, Haller JF, Gerez de Burgos NM, 
Santomé JA 2000. Presence of a fatty acid-binding protein and lipid stores in flight muscle of Dipetalogaster maximus (Hemiptera:Reduviidae). J Med Entomol 37: 938-944.

Chino H 1997. Physiological significance of lipid transport by lipophorin for long-distance flight in insects. Comp Biochem Physiol 117B: 455-461.

Chino H, Kiyomoto Y, Takahashi K 1989. In vitro study of the action of adipokinetic hormone in locusts. $J$ Lipid Res 30: 571-578.

Chino H, Lum PY, Nagao ET, Hiraoka T 1992. The molecular and metabolic essentials for long-distance flight in insects. J Comp Physiol B 162: 101-106.

Forte TM, Nordhausen RW 1986. Electron microscopy of negatively stained lipoproteins. Meth Enzymol 128A: 442457.

Frings CS, Dunn RT 1970. A colorimetric method for determination of total serum lipids based on the sulphophospho-vanillin reaction. Amer J Pathol 3: 89-91.

Gäde G, Auerswald L 2002. Beetles' choice-proline for energy output: control by AKHs. Comp Biochem Physiol 132B: 117-129.

Goldsworthy GJ, Lee MJ, Luswata R, Drake AF, Hyde D 1997. Structures, assays and receptors for locust adipokinetic hormones. Comp Biochem Physiol 117B: 483496.

Gringorten JL, Friend WG 1979. Wing-beat pattern in Rhodnius prolixus Stäl (Heteroptera:Reduviidae) during exhaustive flight. Can J Zool 57: 391-395.

Guzman-Bracho C 2001. Epidemiology of Chagas disease in Mexico: an update. Trends Parasitol 17: 372-376.

Jutsum AR, Goldsworthy GJ 1976. Fuels for flight in Locusta. J Insect Physiol 22: 243-249.

Lehane MJ, Schofield CJ 1982. Flight initiation in Triatoma infestans (Klug) (Hemiptera: Reduviidae). Bull Entomol Res 72: 497-510.

Lum PY, Chino H 1990. Primary role of adipokinetic hormone in the formation of low density lipophorin in locusts. $J$ Lipid Res 31: 2039-2044.

Neghme A, Schenone H, Reyes H, Carrasco J, Alfaro E 1960. Hallazgo de Triatoma infestans en vagones de ferrocaril. Bol Chil Parasitol 15: 86-87.

Noireau F, Dujardin JP 2001. Flight and nutritional status of sylvatic Triatoma sordida and Triatoma guasayana. Mem Inst Oswaldo Cruz 96: 385-389.
Ryan RO, Van der Horst DJ 2000. Lipid transport biochemistry and its role in energy production. Annu Rev Entomol 45: 233-260.

Schofield CJ 1980. Nutritional status of domestic populations of Triatoma infestans. Trans R Soc Trop Med Hyg 74: 772778.

Schofield CJ 1994. Triatominae: Biology \& Control, Eurocommunica Publications, Bognor Regis, UK, 80 pp.

Schofield CJ, Lehane MJ, McEwen P, Catalá SS, Gorla DE 1992. Dispersive flight by Triatoma infestans under natural climatic conditions in Argentina. Med Vet Entomol 6: 51-56.

Scott A, Melvin HE 1953. Determination of dextran with anthrone. Anal Chem 25: 1656-1661.

Sjogren RD, Ryckman RE 1966. Epizootiology of Trypanosoma cruzi in southwestern North America. Part VIII: nocturnal flights of Triatoma proctata (Uhler) as indicated by collections at black light traps (Hemiptera: Reduviidaee: Triatominae). J Med Entomol 3: 81-92.

Soler CA, Schenone H, Reyes H 1969. Problemas derivados de la reaparición de Triatoma infestans en viviendas desinsectadas y el concepto de reinfestación. Bol Chil Parasitol 24: 83-87.

Van Handel E 1965. Microseparation of glycogen, sugars, and lipids. Anal Biochem 11: 266-271.

Van Heusden MC, Van der Horst DJ, Voshol J, Beenakkers AM 1984. In vitro studies on hormone-stimulated lipid mobilization from fat body and interconversion of hemolymph lipoproteins of Locusta migratoria. $J$ Insect Physiol 30: 685-693.

Van Heusden MC, Van der Horst DJ, Voshol J, Beenakkers AM 1987. The recycling of protein components of the flightspecific lipophorin in Locusta migratoria. Insect Biochem 17: 771-776.

Van der Horst DJ, Ryan RO, Van Heusden MC, Schultz TKF, Van Doorn JM, Law JH, Beenakkers AM 1988. An insect lipophorin hybrid helps to define the role of apolipophorinIII. J Biol Chem 263: 2027-2033.

Ward JP, Candy DJ, Smith SN 1982. Lipid storage and changes during flight by Triatominae bugs (Rhodnius prolixus and Triatoma infestans). J Insect Physiol 28: 527-534.

WHO-World Health Organization 1991. Control of Chagas Disease, Technical Report Series, 811, Geneva, 91 pp.

Wyatt GR 1967. The biochemistry of sugars and polysaccharides in insects. Adv Insect Physiol 4: 287-360. 